\title{
Age at Menarche, Duration of Monthly Flow and Cycle Length as Risk Factors for Endometriosis in Sub-Sahara Black African Women
}

\author{
Abayomi B Ajayi, ${ }^{1}$ Bamgboye M Afolabi, ${ }^{2,3 *}$ Victor D Ajayi, ${ }^{1}$ Ifeoluwa Oyetunji, ${ }^{1}$ Adedamilola Atiba ${ }^{1}$ \\ ${ }^{1}$ Nordica Fertility Center, Nigeria \\ ${ }^{2}$ Health, Environment and Development Foundation, Nigeria \\ ${ }^{3}$ African, Pan-African Health Alliance and Collaborative, Nigeria
}

\begin{abstract}
Introduction: Endometriosis is an enigmatic and multifaceted ailment that distresses a large number of women worldwide which may trigger pain, infertility and other discomforts such as dysmenorrhea.

Materials and Methods: Data from the medical records of 113 women with and 113 age-matched women without endometriosis were analyzed retrospectively to investigate association of three domains of menstrual characteristics - age at menarche, duration of menstrual flow and cycle length - with risk of developing endometriosis. STATA 13 statistical software was used for analysis of data.

Results: Sub-fertile women with menarche at $<11$ years were 1.80 times more likely to have endometriosis (OR=1.80, 95\% CI: $0.51,6.33$ ) and had 1.29 times the risk of the disease ( $R R=1.29,95 \% \mathrm{CI}: 0.81,2.06)$ influenced by body mass index $\left(\mathrm{BMI} \mathrm{in} \mathrm{Kg} / \mathrm{m}^{2}\right)$. Women with duration of flow of 4-7 days were 2.33 times more likely to have endometriosis. Women with cycle length of $\geq 28$ days were 1.45 times more likely to also have endometriosis. Mean egg yield at OPU from endometriotic women was significantly lower than that from women without endometriosis when age at menarche was $\geq 14$ years ( $\mathrm{t}$-test $=-4.44$, P-value $=0.00000001$ ), when duration of flow (days) was 4-7 ( $\mathrm{t}$-test $=-4.78, \mathrm{P}-\mathrm{value}=0.00000001$ ) and when cycle length was $\geq 28$ days ( $\mathrm{t}$-test $=-4.35$, P-value $=0.00000001$ ). The mean matured eggs (MII) was very significantly higher when age at menarche was $\geq 14$ years ( $\mathrm{t}$-test $=-2.59$, P-value $=0.006$ ), when duration of flow was 4-7 days ( $\mathrm{t}$-test $=2.81$, $\mathrm{P}$-value $=0.003$ ) and when the cycle length was $\geq 28$ days ( $\mathrm{t}$-test $=-2.79$, $\mathrm{P}$-value $=0.003$ ). Multivariate regression analysis indicated that menarche, number of days of menstrual flow and cycle length gave a significant $90 \%$ of the variation found in endometriosis $(\mathrm{R} 2=0.9001, \mathrm{~F}=648.77$, $\mathrm{P}$-value $=0.000000001)$.
\end{abstract}

Conclusion: Menarche at age $<11$ years and cycle length of $\geq 28$ days were significantly associated with and were better predictors of laparoscopic ally confirmed endometriosis. BMI influenced all of the three domains of menarche, duration of flow (days) and cycle length. Endometriotic women produced fewer oocytes but fertilization rate was not compromised.

Keywords: Endometriosis, Menstrual characteristics, Dysmenorrhea, Egg yield at ovum pick up (OPU), Sub-sahara african women, Infertility

Abbreviations: APAHAC: african, pan-african health alliance and collaborative; BMI: body mass index; CI: confidence interval; Coef: coefficient; MII: Eggs in the second phase of meiosis and are mature; NFC: nordica fertility center; OPU: ovum pickup; OR: odds ratio; PID: pelvic inflammatory disease; RR: risk ratio; SSA: sub-sahara africa; Std Err: standard error; WHO: world health organization

\section{Introduction}

Controversy still surrounds the issue of age at menarche and cycle length as risk factors for endometriosis a disease that has been well studied over the years but still an enigma till today. It is a very common ailment occurring in $6-10 \%$ of the general female population; in women with pain, infertility or both, with a frequency of 35-50\%. ${ }^{1}$ Endometriosis is the presence of endometrial-like tissue
Quick Response Code:

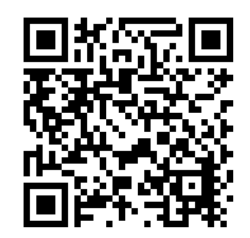

\begin{tabular}{|c|c|}
\hline \multicolumn{2}{|c|}{$\begin{array}{l}\text { *Corresponding author: Bamgboye M Afolabi, 18, Ogunfunmi Street, Off Akobi Crescent, } \\
\text { Surulere, Lagos, Nigeria }\end{array}$} \\
\hline Received: 20 March, 2021 & Published: 16 April, 2021 \\
\hline $\begin{array}{l}\text { Citation: Ajayi AB, Afolabi } \mathrm{E} \\
\text { y Flow and Cycle Length as } \\
\text { Pregn Womens Health Care }\end{array}$ & $\begin{array}{l}\text { yetunji I, Atiba A. Age at Menarche, Duration of Mon } \\
\text { on Endometriosis in Sub-Sahara Black African Wom } \\
\text { ):1-12. DOI: 10.53902/PWHCIJ.2021.01.000505 }\end{array}$ \\
\hline
\end{tabular}


(glands and stroma) outside the uterus that induces a chronic inflammatory reaction, scar tissue and adhesions that may distort a woman's pelvic anatomy. ${ }^{2}$ In this disorder, the ectopic endometrial tissue is non-malignant, proliferative and is diagnosed by laparoscopic observation of lesions, implants, nodules and cysts on the pelvic peritoneum, the pelvic organs, ${ }^{3,4}$ or other parts of the body such as the uterosacral ligament, retro-cervical area, rectovaginal septum, rectum sigmoid colon, terminal ileum, vermiform appendix, bladder and ureter. ${ }^{5-9}$ Studies have suggested early age at menarche as a risk factor for endometriosis,,$^{10,11}$ and that menarche at 14 years or after diminishes the risk of endometriosis. ${ }^{12,13}$ Matalliotakis et al., ${ }^{14}$ suggested that menstrual cycle characteristics such as cycle length, may be related to endometriosis incidence while others had a different opinion. ${ }^{15-17}$ The prevalence and risk-factors of endometriosis are abundantly documented among women in developed world, though data on this ailment is still rare among indigenous sub-Saharan Black African women. The present study assesses the association or otherwise between menarche, days of menstrual flow and cycle length on one part and the probability of endometriosis on another part. This study is important in view of growing attention from patients, clinicians, embryologists and scientists in sub-Saharan Africa, to improve awareness, diagnosis and treatment of endometriosis. ${ }^{18}$ The goal of this study is to provide further information on endometriosis and its risk factors among Black African women. The objective is to determine the menstrual characteristics that associate more with endometriosis among this group of women.

\section{Materials and Methods}

This retrospective case-control study of sub-fertile women with and without endometriosis was conducted from July to December 2019 at the Nordica Fertility Center (NFC), Lagos, Nigeria. The Materials and Methods had earlier been described ${ }^{19}$ but is briefly summarized below. During the period of study, the medical records of all patients who presented since 2010 with a history of infertility (either primary or secondary), pelvic pain, painful menstruation and/or excessive menstrual bleeding documented relevant information concerning their socio-economic profile, medical, gynecological and obstetric history, and also rendered indications on medication use, family history and other vital information. The attending gynecologist recorded patients' symptomatology of endometriosis such as dysmenorrhea, dyspareunia, bleeding from other sites concurrently with monthly menstruation and if other siblings or family member experienced such symptoms. The gynecologist also inquired and documented age at menarche, duration monthly menstruation and cycle length. Patients with provisional finding of endometriosis underwent ultrasound imaging followed by laparoscopic examination to determine a conclusive diagnosis of endometriosis and the stage of the disease. Laparoscopic surgical procedures, using standard equipment, were mandatorily under general anesthesia in the endoscopy unit of Nordica Fertility Center, Lagos. Images were shown on a high definition screen using a 3D-chip camera. Laparoscopy with multiple-port access was the main surgery performed and consequently diagnosis, staging, retrieval of sample for histological examination and diagnosis, adhesiolysis and ablative surgery were performed as required, by experiences surgical team. Those who served as control were women who presented primarily with infertility but without symptomatology of endometriosis. The socio-demographic, medical, obstetric and gynecological, family and medication history were also collected from those without the diagnosis of endometriosis. Thus, all patients who had a laparoscopic and histological diagnosis and staging of endometriosis and who consented were included in the study as cases while those who consulted primarily for infertility was included as control group. All consenting study subjects were indigenous Black African women of Nigerian origin. Excluded from the study were

(i) Caucasian and non-Nigerian patients

(ii) Those who did not consent to the study

(iii) patients who were severely anemic

(iv) Those who had other gynecological problems apart from endometriosis and infertility

Sample size determination: Sample size was calculated using power analysis program (PASS2020 Utah, USA) and two-sample t-tests assuming equal variance. With conditional power set at 0.9 and Alpha set at 0.025 and assuming equal variance, the calculated sample size was 101 , which indicated, through futility analysis result of $0.2(<0.8)$, that the study should continue because the calculated sample size was large enough to achieve statistical significance. The sample size was however further inflated by $10 \%$ to account for "drop-outs" (patients with incomplete data, since this was a retrospective study) and rounding up the total sample size to 113 . Age-matched control subjects of the same sample size were selected.

Consent of patients: All patients consulting at Nordica Fertility Center were informed that their data may be used for teaching and research purposes. All patients in this study were part of those who were informed about the study and the purpose for it and all gave verbal approval for their data to be used in the study. All patients were assured of data confidentiality, non-use of names of subjects and safe keeping of their medical records. Data of patients who did not give consent were removed from the study and therefore not included in analysis and presentation.

Ethical consideration: This study was approved by the Nigerian Institute of Medical Research Review Board (NIMR-IRB-18-006)

Data analysis: Body Mass Index (BMI) in $\mathrm{Kg} / \mathrm{m}^{2}$ was classified according to WHO classification ${ }^{20}$ as underweight $(<18.5)$, normal weight (18.5-24.9), overweight (25.0-29.9) and obese $(\geq 30)$. Primary infertility refers to a woman who had never conceived and secondary infertility to a woman who had ever been pregnant. Data management, including entry, coding, and cleaning was conducted in Excel spreadsheet and exported into STATA 13 (StataCorp, Texas 77845, USA) prior to statistical analysis. Chi-square, Odds Ratio (OR) Risk Ratio (RR) with their 95\% confidence interval (95\% $\mathrm{CI})$ and Student's t-test were used to analyze association between different variables. If OR is $>1$, and the $95 \%$ CI do not include 1 , it was assumed that there is a statistical association, with $95 \%$ con- 
fidence, between the disease and the exposure. ${ }^{15}$ The patient's age, parity age of menarche, the length of the menstrual cycles and the duration of menstrual flow were documented of each case or control recruited were recorded. The factors considered in this study to increase the occurrence of endometriosis, were (i) menarche at an age $<11$ years, (ii) duration of menstruation $>7$ days, and (iii) menstrual cycle length $<28$ days. Odds ratio (OR), which compares the probability that sub-fertile women with (cases) and without (control) endometriosis were exposed to the same menstrual risk factors, was used to determine the association between the presence of certain menstrual characteristics and the occurrence of endometriosis. Multivariate regression analysis was used to further ascertain association between dependent and independent vari- ables. P-value $<0.05$ was taken as statistically significant. Data were presented mainly as Tables.

\section{Results}

Demographic, menstrual and fertility characteristics of study subjects (Table 1). The demographic and fertility profile of the study subjects are as illustrated in Table 1. The endometriosis group was matched on age, duration of infertility, In-vitro fertilization (IVF) treatment cycles attempted, duration of flow and cycle length but not on body mass index ( $\mathrm{t}$-test $=-3.44$, $\mathrm{P}$-value=0.0004), parity ( $\mathrm{t}$-test $=-1.90, \mathrm{P}$-value $=0.03$ ) and menarche ( $\mathrm{t}$-test $=-2.16$, $\mathrm{P}$-value $=0.03$ ) with the no-endometriosis group.

Table 1: Demographic and fertility profile of women in the study.

\begin{tabular}{|c|c|c|c|c|c|c|}
\hline \multirow{2}{*}{ Variable } & \multirow{2}{*}{ Statistics } & All & Endometriosis & No endometriosis & \multirow{2}{*}{ t-test } & \multirow{2}{*}{ P-value } \\
\hline & & $n=226(100.0 \%)$ & $n=113(50.0 \%)$ & $n=113(50.0 \%)$ & & \\
\hline \multirow{3}{*}{ Age (years) } & Mean & 34.30 & 33.93 & 34.61 & \multirow{2}{*}{-1.05} & \multirow{2}{*}{0.15} \\
\hline & \pm sd & 4.9 & 4.3 & 5.4 & & \\
\hline & Range & $21-49$ & $25-49$ & $21-47$ & & \\
\hline \multirow{3}{*}{ BMI $\left(\mathrm{kg} / \mathrm{m}^{2}\right)$} & Mean & 27.01 & 25.80 & 27.95 & \multirow{2}{*}{-3.44} & \multirow{2}{*}{0.0004} \\
\hline & \pm sd & 4.8 & 4.9 & 4.5 & & \\
\hline & Range & $17.1-44.0$ & $17.1-42.0$ & $20.0-44.0$ & & \\
\hline \multirow{2}{*}{ Parity } & Median & 0 & 0 & 0 & - & - \\
\hline & Range & $0-3$ & $0-1$ & $0-3$ & \multicolumn{2}{|c|}{-} \\
\hline \multirow{3}{*}{$\begin{array}{l}\text { Duration of infertil- } \\
\text { ity (years) }\end{array}$} & Mean & 4.93 & 4.63 & 5.18 & \multirow{2}{*}{-1.12} & \multirow{2}{*}{0.13} \\
\hline & $\pm \mathrm{sd}$ & 3.7 & 3.2 & 4.1 & & \\
\hline & Range & $0-29$ & $0.4-20$ & $0-29$ & & \\
\hline \multirow{3}{*}{$\begin{array}{l}\text { IVF treatments } \\
\text { cycles attempted }\end{array}$} & Mean & 1.22 & 1.24 & 1.20 & \multirow{2}{*}{0.41} & \multirow{2}{*}{0.34} \\
\hline & $\pm \mathrm{sd}$ & 0.7 & 0.9 & 0.5 & & \\
\hline & Range & $0-6$ & $0-6$ & $1-3$ & & \\
\hline \multirow{3}{*}{$\begin{array}{c}\text { Age at menarche } \\
\text { (years) }\end{array}$} & Mean & 13.11 & 12.88 & 13.34 & \multirow{2}{*}{-2.16} & \multirow{2}{*}{0.02} \\
\hline & $\pm \mathrm{sd}$ & 1.6 & 1.6 & 1.6 & & \\
\hline & Range & 8-18 & $8-18$ & $9-17$ & & \\
\hline \multirow{3}{*}{$\begin{array}{l}\text { Duration of flow } \\
\text { (days) }\end{array}$} & Mean & 4.82 & 4.90 & 4.74 & \multirow{2}{*}{0.91} & \multirow{2}{*}{0.18} \\
\hline & $\pm s d$ & 1.3 & 1.1 & 1.5 & & \\
\hline & Range & $2-10$ & $2-9$ & $2-10$ & & \\
\hline \multirow{3}{*}{ Cycle length (days) } & Mean & 29.99 & 29.55 & 30.4 & \multirow{2}{*}{-1.00} & \multirow{2}{*}{0.16} \\
\hline & \pm sd & 6.7 & 3.44 & 8.8 & & \\
\hline & Range & $21-90$ & $23-45$ & $21-90$ & & \\
\hline
\end{tabular}

Age at menarche and probability of endometriosis (Table 2). Table 2 compares the odds ratio and risk ratio for overall age-specific menarche and BMI-stratified menarche women with and without endometriosis. The probability of endometriosis was highest $(\mathrm{OR}=1.80,95 \% \mathrm{CI}: 0.51,6.33)$ when menarche at age $<11$ years than when age of menarche was 11.13 .9 years $(\mathrm{OR}=1.61,95 \%$ $\mathrm{CI}: 0.94,2.74)$. The chance of endometriosis was very low $(\mathrm{OR}=0.54$, $95 \%$ CI: $0.31,0.94$ ) when age at menarche was $\geq 14$ years. This suggest that among Black African women, menarche at age $<11$ years was more related to endometriosis, though some women could like develop endometriosis when menarche was $11-13.9$ years. Re- gardless of the age at menarche, the risk of endometriosis was high (RR=1.62, 95\% CI: 1.35, 1.94) among women with BMI $<18.5 \mathrm{Kg} /$ $\mathrm{m}^{2}$. Among normal weight women, the probability of endometriosis was higher (OR=1.26, 95\% CI: $0.49,3.20$ ) among those who had menarche at 11-13.9 years than among those who had menarche at $\geq 14$ years (OR=0.56, 95\% CI: $0.22,1.45$ ). Overweight women were 2.81 as likely to present with endometriosis when they had menarche at age $<11$ years (OR=2.81, 95\% CI: $0.25,32.16)$ than those who had menarche at age 11-13.9 years $(\mathrm{OR}=1.79,95 \% \mathrm{CI}: 0.76$, 4.21). A very low probability of endometriosis was observed when age at menarche was $\geq 14$ years. Obese women with menarche at 
age 11-13.9 years were 2.33 times more likely to have endometriosis (OR=2.33, 95\% CI: $0.72,7.60$ ) than those who had early or late age at menarche.

Duration of flow and probability of endometriosis (Table 2). Overall, regardless of the BMI, the duration of menstrual flow did not seem to predict the probability of endometriosis as those who had 4-7 day so of menstrual flow were 2.33 times more likely to have endometriosis (OR=2.33, 95\% CI: 1.15, 4.71), which appeared to be driven by overweight women (OR=3.14, 95\% CI: $0.94,10.44$ ) than by normal weight women $(\mathrm{OR}=2.39,95 \% \mathrm{CI}: 0.71,8.00)$ or by obese women (OR=1.59, 95\% CI: 0.36, 7.01).

Table 2: Probability and risk of endometriosis relative to menstrual characteristics.

\begin{tabular}{|c|c|c|c|c|c|c|c|c|c|c|c|c|c|c|c|}
\hline \multirow{2}{*}{$\begin{array}{l}\text { Vari- } \\
\text { able }\end{array}$} & \multirow{2}{*}{ Item } & \multicolumn{4}{|c|}{ Endometriosis } & \multicolumn{4}{|c|}{ No endometriosis } & \multirow{2}{*}{ t-test } & \multirow{2}{*}{$\begin{array}{c}\text { P-val- } \\
\text { ue }\end{array}$} & \multirow{2}{*}{$\chi^{2}$} & \multirow{2}{*}{$\begin{array}{c}\text { P-val- } \\
\text { ue }\end{array}$} & \multirow{2}{*}{$\begin{array}{l}\text { OR (95\% } \\
\text { CI) }\end{array}$} & \multirow{2}{*}{$\begin{array}{c}\text { RR }(95 \% \\
\text { CI) }\end{array}$} \\
\hline & & Freq. & $\%$ & Mean & $\pm s d$ & Freq. & $\%$ & Mean & $\pm s d$ & & & & & & \\
\hline \multirow{20}{*}{$\begin{array}{l}\text { Age at } \\
\text { men- } \\
\text { arche } \\
\text { (yrs.) }\end{array}$} & \multicolumn{15}{|c|}{ All } \\
\hline & $<11$ & 7 & 6.19 & 9.71 & 0.76 & 4 & 3.54 & 9.25 & 0.50 & 1.21 & 0.13 & 0.38 & 0.54 & $\begin{array}{c}1.80(0.51 \\
6.33)\end{array}$ & $\begin{array}{c}1.29(0.81 \\
2.06)\end{array}$ \\
\hline & $\begin{array}{l}11- \\
13.9\end{array}$ & 72 & 63.72 & 12.33 & 0.77 & 59 & 52.21 & 12.37 & 0.67 & -0.32 & 0.38 & 3.06 & 0.08 & $\begin{array}{c}1.61(0.94 \\
2.74)\end{array}$ & $\begin{array}{c}1.27(0.96 \\
1.68)\end{array}$ \\
\hline & $\geq 14.0$ & 34 & 30.09 & 14.68 & 0.91 & 50 & 44.25 & 14.80 & 0.88 & -0.60 & 0.27 & 4.83 & 0.03 & $\begin{array}{c}0.54(0.31 \\
0.94)\end{array}$ & $\begin{array}{c}0.73(0.54 \\
0.98)\end{array}$ \\
\hline & \multicolumn{15}{|c|}{ For BMI $<18.5$} \\
\hline & $<11$ & 0 & 0.0 & 0.0 & 0.0 & 0 & 0.0 & 0.0 & 0.0 & - & - & 1.04 & 0.31 & Undefined & $\begin{array}{c}1.62(1.35, \\
1.94)\end{array}$ \\
\hline & $\begin{array}{l}11- \\
13.9\end{array}$ & 3 & 60.0 & 11.67 & 1.15 & 0 & 0.0 & 0.0 & 0.0 & - & - & 1.04 & 0.31 & Undefined & $\begin{array}{c}1.62(1.35 \\
1.94)\end{array}$ \\
\hline & $\geq 14.0$ & 2 & 40.0 & 15.00 & 0.0 & 0 & 0.0 & 0.0 & 0.0 & - & - & 1.04 & 0.31 & Undefined & $\begin{array}{c}1.62(1.35 \\
1.94)\end{array}$ \\
\hline & \multicolumn{15}{|c|}{ For BMI 18.5-24.9 } \\
\hline & $<11$ & 4 & 8.16 & 10.0 & 0.0 & 0 & 0.0 & 0.0 & 0.0 & - & - & 1.04 & 0.31 & Undefined & $\begin{array}{c}1.62(1.35 \\
1.94)\end{array}$ \\
\hline & $\begin{array}{c}11- \\
13.9\end{array}$ & 29 & 59.19 & 12.31 & 0.76 & 15 & 53.57 & 12.33 & 0.62 & -0.09 & 0.47 & 0.23 & 0.63 & $\begin{array}{c}1.26(0.49 \\
3.20)\end{array}$ & $\begin{array}{c}1.09(0.77 \\
1.54)\end{array}$ \\
\hline & $\geq 14.0$ & 16 & 32.65 & 14.38 & 0.62 & 13 & 46.43 & 15.00 & 0.82 & -2.25 & 0.02 & 1.42 & 0.23 & $\begin{array}{c}0.56(0.22 \\
1.45)\end{array}$ & $\begin{array}{c}0.80(0.55 \\
1.17)\end{array}$ \\
\hline & \multicolumn{15}{|c|}{ For BMI 25.0-29.9 } \\
\hline & $<11$ & 2 & 5.13 & 9.0 & 1.41 & 1 & 1.89 & 10.0 & 0.0 & - & - & 0.07 & 0.78 & $\begin{array}{c}2.81(0.25 \\
32.16)\end{array}$ & $\begin{array}{c}1.60(0.69 \\
3.70)\end{array}$ \\
\hline & $\begin{array}{l}11- \\
13.9\end{array}$ & 26 & 66.67 & 12.31 & 0.79 & 28 & 52.83 & 12.39 & 0.69 & -0.40 & 0.35 & 1.75 & 0.19 & $\begin{array}{c}1.79(0.76 \\
4.21)\end{array}$ & $\begin{array}{c}1.41(0.84 \\
2.37)\end{array}$ \\
\hline & $\geq 14.0$ & 11 & 28.20 & 14.91 & 0.83 & 24 & 45.28 & 14.79 & 0.83 & 0.40 & 0.65 & 2.75 & 0.10 & $\begin{array}{c}0.47(0.20 \\
1.15)\end{array}$ & $\begin{array}{c}0.64(0.37 \\
1.16)\end{array}$ \\
\hline & \multicolumn{15}{|c|}{ For $\mathrm{BMI} \geq 30.0$} \\
\hline & $<11$ & 1 & 5.00 & 10.00 & 0.00 & 3 & 9.38 & 9.00 & 0.00 & - & - & 0.00 & 0.97 & $\begin{array}{c}0.51(0.05 \\
5.26)\end{array}$ & $\begin{array}{c}0.63(0.11 \\
3.57)\end{array}$ \\
\hline & $\begin{array}{l}11- \\
13.9\end{array}$ & 14 & 70.00 & 12.57 & 0.65 & 16 & 50.00 & 12.38 & 0.72 & 0.76 & 0.23 & 1.98 & 0.16 & $\begin{array}{c}2.33(0.72 \\
7.60)\end{array}$ & $\begin{array}{c}1.71(0.78 \\
3.74)\end{array}$ \\
\hline & $\geq 14.0$ & 5 & 25.00 & 15.00 & 1.73 & 13 & 40.62 & 14.62 & 1.04 & 0.46 & 0.33 & 0.73 & 0.39 & $\begin{array}{c}0.49(0.14 \\
1.67)\end{array}$ & $\begin{array}{c}0.63(0.27 \\
1.45)\end{array}$ \\
\hline
\end{tabular}




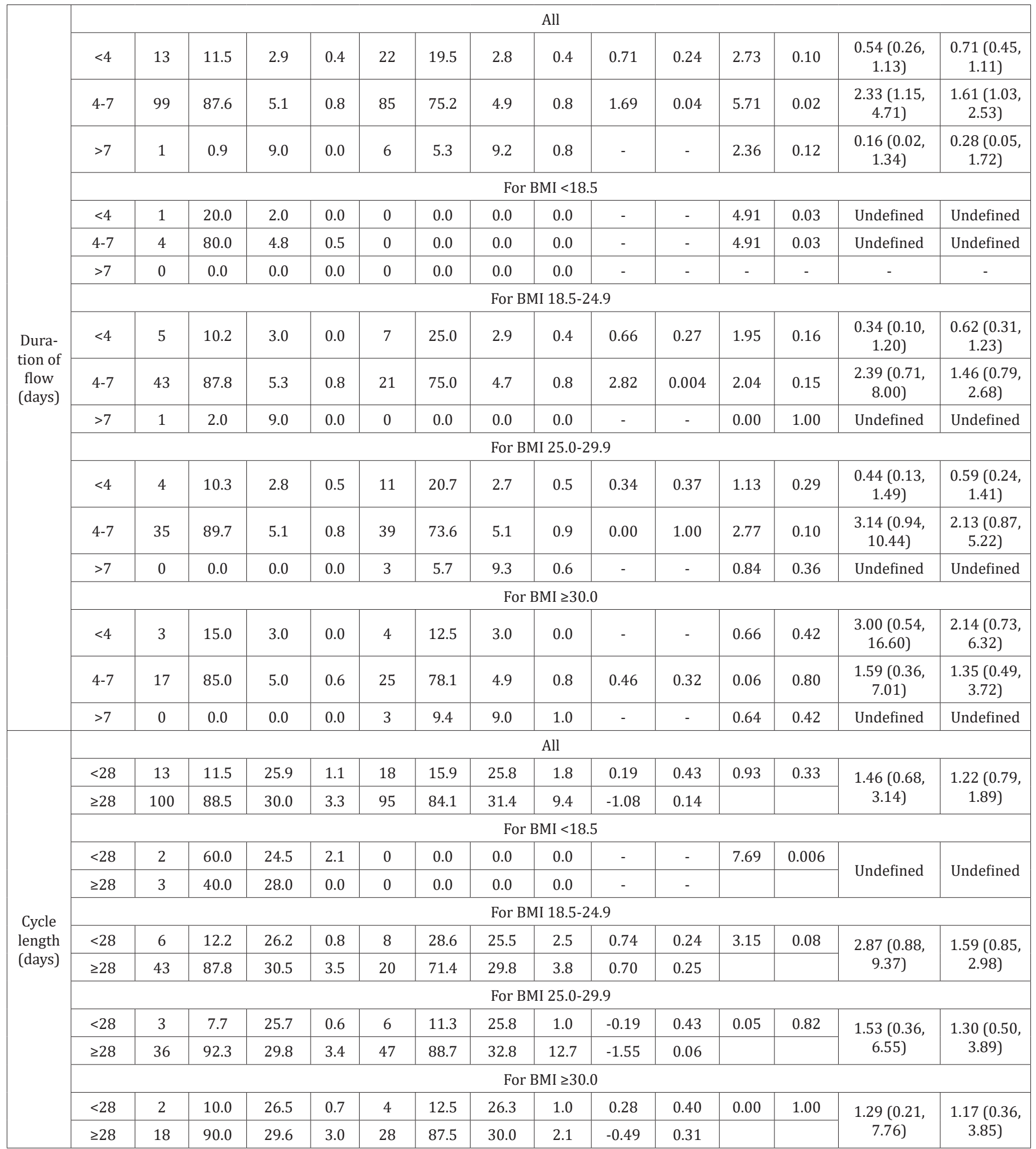

Cycle length and probability of endometriosis (Table 2). Normal weight women with cycle length $\geq 28$ days were 2.87 times more likely to have endometriosis $\left(\chi^{2}=3.15\right.$, P-value $=0.08,0 R=2.87,95 \%$ CI: $0.88,9.37)$ than those with cycle length $<28$ days. However, overweight women with cycle length $\geq 28$ days were only 1.53 times more likely to have endometriosis $\left(\chi^{2}=0.05, \mathrm{P}\right.$-value $=0.82, \mathrm{OR}=1.53$, $95 \%$ CI: $0.36,6.55)$ than those with cycle length <28 days, whereas obese women with cycle length $\geq 28$ days were only 1.29 times more likely to have endometriosis $\left(\chi^{2}=0.00, \mathrm{P}\right.$-value=1.00, $\mathrm{OR}=1.29$, $95 \%$ CI: $0.21,7.76$ ) than those with cycle length $<28$ days. Of all the three categories of BMI it is, apparently, those with normal weight and cycle length $\geq 28$ days that are most probable to present with endometriosis.

Parity, duration and type of infertility by menstrual characteristics relative to probability of endometriosis (Table 3). 
Table 3: Parity, duration and type of infertility by menstrual characteristics and probability of endometriosis.

\begin{tabular}{|c|c|c|c|c|c|c|c|c|c|c|c|c|c|c|c|c|c|c|c|}
\hline \multirow{5}{*}{\multicolumn{2}{|c|}{ Variable }} & \multicolumn{6}{|c|}{ Age at menarche $<11 \mathrm{yrs}$. } & \multicolumn{6}{|c|}{ Duration of flow $<4$ days } & \multicolumn{6}{|c|}{ Cycle length $<28$ days } \\
\hline & & \multicolumn{6}{|c|}{ OR $(95 \% \mathrm{CI})=1.80(0.51,6.33)$} & \multicolumn{6}{|c|}{ OR $(95 \%$ CI $)=0.54(0.26,1.13)$} & \multicolumn{6}{|c|}{ OR $(95 \% \mathrm{CI})=1.45(0.68,3.14)$} \\
\hline & & \multirow{2}{*}{\multicolumn{2}{|c|}{ Parity }} & \multirow{2}{*}{\multicolumn{2}{|c|}{$\begin{array}{l}\text { Duration of } \\
\text { infertility }\end{array}$}} & \multicolumn{2}{|c|}{$\begin{array}{c}\text { Type of infer- } \\
\text { tility }\end{array}$} & \multirow{2}{*}{\multicolumn{2}{|c|}{ Parity }} & \multirow{2}{*}{\multicolumn{2}{|c|}{$\begin{array}{c}\text { Duration of } \\
\text { infertility }\end{array}$}} & \multicolumn{2}{|c|}{$\begin{array}{c}\text { Type of } \\
\text { infertility }\end{array}$} & \multirow{2}{*}{\multicolumn{2}{|c|}{ Parity }} & \multirow{2}{*}{\multicolumn{2}{|c|}{$\begin{array}{l}\text { Duration of } \\
\text { infertility }\end{array}$}} & \multicolumn{2}{|c|}{$\begin{array}{c}\text { Type of infer- } \\
\text { tility }\end{array}$} \\
\hline & & & & & & \multirow{2}{*}{\begin{tabular}{|c|}
$1^{\mathbf{o}}$ \\
$\begin{array}{c}\text { Freq. } \\
(\%)\end{array}$ \\
\end{tabular}} & \multirow{2}{*}{\begin{tabular}{|c|}
$2^{\circ}$ \\
$\begin{array}{c}\text { Freq. } \\
(\%)\end{array}$ \\
\end{tabular}} & & & & & \multirow{2}{*}{\begin{tabular}{|c|}
$1^{\circ}$ \\
$\begin{array}{c}\text { Freq. } \\
(\%)\end{array}$ \\
\end{tabular}} & \multirow{2}{*}{$\begin{array}{c}2^{\circ} \\
\text { Freq. } \\
(\%)\end{array}$} & & & & & $\mathbf{1}^{\mathbf{0}}$ & $2^{\circ}$ \\
\hline & & Mean & $\pm s d$ & Mean & $\pm s d$ & & & Mean & $\pm s d$ & Mean & $\pm s d$ & & & Mean & $\pm s d$ & Mean & $\pm s d$ & $\begin{array}{l}\text { Freq. } \\
\text { (\%) }\end{array}$ & $\begin{array}{l}\text { Freq. } \\
(\%)\end{array}$ \\
\hline \multicolumn{2}{|c|}{ Endometriosis } & 0.14 & 0.4 & 4.14 & 2.34 & $5(62.5)$ & $\begin{array}{c}2 \\
(66.7)\end{array}$ & 0.31 & 0.5 & 6.04 & 3.2 & 5 & 8 & 0.23 & 0.4 & 4.22 & 3.0 & $\begin{array}{c}8 \\
(42.1)\end{array}$ & $\begin{array}{c}5 \\
(41.7)\end{array}$ \\
\hline \multicolumn{2}{|c|}{$\begin{array}{l}\text { No endome- } \\
\text { triosis }\end{array}$} & 0.0 & 0.0 & 4.25 & 4.57 & $3(37.5)$ & $\begin{array}{c}1 \\
(33.3)\end{array}$ & 0.23 & 0.4 & 5.58 & 3.9 & 6 & 16 & 0.16 & 0.4 & 6.59 & 6.2 & $\begin{array}{c}11 \\
(57.9)\end{array}$ & $\begin{array}{c}7 \\
(58.3)\end{array}$ \\
\hline \multirow{4}{*}{$\begin{array}{l}\text { Sta- } \\
\text { tis- } \\
\text { tics }\end{array}$} & t-test & \multicolumn{2}{|c|}{ - } & \multicolumn{2}{|c|}{-0.04} & - & - & \multicolumn{2}{|c|}{0.49} & 0. & & & - & 0.4 & & -1.4 & & 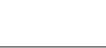 & \\
\hline & $\begin{array}{c}\text { P-val- } \\
\text { ue }\end{array}$ & - & & 0. & & - & - & 0.3 & & 0. & & & & 0.3 & & 0.0 & & & \\
\hline & $\begin{array}{c}\chi^{2} \\
\text { (P-val- } \\
\text { ue) }\end{array}$ & - & & 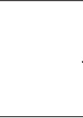 & & 0.00( & $.00)$ & & & - & & 0.10 & $(0.75)$ & - & & - & & 0.00 & $1.00)$ \\
\hline & $\begin{array}{c}\text { OR } \\
(95 \% \\
\text { CI) } \\
\end{array}$ & - & & . & & $\begin{array}{r}0.83( \\
13.6\end{array}$ & $\begin{array}{l}.05 \\
3)\end{array}$ & & & - & & $\begin{array}{r}1.6 \\
7\end{array}$ & $\begin{array}{l}(0.39, \\
17)\end{array}$ & - & & & & $1.02(0$. & $4,4.41)$ \\
\hline & & & Age a & menar & $h e=11$ & - 13.9 yrs & & & Dur & ation of & low 4- & 7 days & & & & cle leng & th $\geq 28$ & days & \\
\hline & & & OR & $\% \mathrm{CI})$ & 1.61( & $.94,2.74)$ & & & $R(95$ & $\% \mathrm{CI})=2$ & 33 (1. & $15,4.7$ & & & OR $(9$ & $\% \mathrm{CI})=$ & $1.27(\mathrm{C}$ & $.73,2.21$ & \\
\hline Endo & etriosis & 0.13 & 0.3 & 4.62 & 3.3 & $\begin{array}{c}46 \\
(63.0) \\
\end{array}$ & $\begin{array}{c}26 \\
(44.8) \\
\end{array}$ & 0.11 & 0.3 & 4.47 & 3.1 & 64 & 35 & 0.12 & 0.3 & 4.68 & 3.2 & $\begin{array}{c}62 \\
(61.4)\end{array}$ & $\begin{array}{c}38 \\
(40.4)\end{array}$ \\
\hline $\begin{array}{r}\text { No e } \\
\mathrm{t}\end{array}$ & $\begin{array}{l}\text { dome- } \\
\text { osis }\end{array}$ & 0.32 & 0.7 & 5.03 & 4.3 & $\begin{array}{c}27 \\
(37.0) \\
\end{array}$ & $\begin{array}{c}32 \\
(55.2) \\
\end{array}$ & 0.26 & 0.6 & 5.03 & 4.2 & 39 & 46 & 0.26 & 0.6 & 4.91 & 3.5 & $\begin{array}{c}39 \\
(38.6) \\
\end{array}$ & $\begin{array}{c}56 \\
(59.6) \\
\end{array}$ \\
\hline & t-test & -1.5 & & -0 & & - & & -2.0 & & -0 . & & & - & -2.0 & & -0.4 & & & \\
\hline & $\begin{array}{c}\text { P-val- } \\
\text { ue }\end{array}$ & 0.0 & & 0. & & - & & 0.0 & & 0. & & & 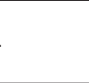 & 0.0 & & 0.3 & & 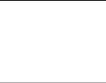 & \\
\hline $\begin{array}{l}\text { Sta- } \\
\text { tis- } \\
\text { tics }\end{array}$ & $\begin{array}{c}\chi^{2} \\
\text { (P-val- } \\
\text { ue) }\end{array}$ & - & & 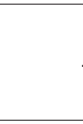 & & 4.290 & .04) & - & & 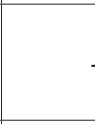 & & 6.50 & $(0.01)$ & - & & - & & 8.52( & $.004)$ \\
\hline & $\begin{array}{c}\text { OR } \\
(95 \% \\
\text { CI }) \\
\end{array}$ & - & & . & & 2.10 & $4,4.23)$ & - & & . & & $\begin{array}{r}2.1 \\
3\end{array}$ & $\begin{array}{l}\text { (1.19, } \\
\text { 90) }\end{array}$ & - & & - & & 2.34 & $2,4.16)$ \\
\hline & & & Age & t mens & che $\geq$ & $14.0 \mathrm{yrs}$. & & & Dur & ation of & low $>$ & days & & & & & & & \\
\hline & & & OR & $\% \mathrm{CI})$ & 0.54( & $.31,0.94)$ & & & $R(95$ & $\% \mathrm{CI})=0$ & $16(0$. & $2,1.3$ & & & & & & & \\
\hline Endo & etriosis & 0.15 & 0.4 & 4.75 & 3.0 & 19 & 15 & 0.0 & 0.0 & 2.00 & 0.0 & 1 & 0 & & & & & & \\
\hline No e & $\begin{array}{l}\text { dome- } \\
\text { osis }\end{array}$ & 0.26 & 0.5 & 5.43 & 3.8 & 20 & 30 & 0.17 & 0.4 & 5.83 & 2.9 & 5 & 1 & & & & & & \\
\hline & t-test & -1.1 & & -0 & & - & & - & & 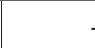 & & & - & & & & & & \\
\hline & $\begin{array}{c}\text { P-val- } \\
\text { ue }\end{array}$ & 0.1 & & 0 . & & - & & - & & . & & & - & & & & & & \\
\hline $\begin{array}{l}\text { Sta- } \\
\text { tis- } \\
\text { tics }\end{array}$ & $\begin{array}{c}\chi^{2} \\
\text { (P-val- } \\
\text { ue) }\end{array}$ & - & & . & & 0.31( & $.58)$ & - & & . & & 0.00 & $(1.00)$ & & & & & & \\
\hline & $\begin{array}{c}\text { OR } \\
(95 \% \\
\text { CI })\end{array}$ & - & & . & & $1.64(0.5$ & $1,5.23)$ & - & & 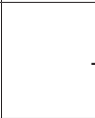 & & Unc & fined & & & & & & \\
\hline
\end{tabular}

Menarche: Only among those who had menarche at 11-13.9 years was there any substantial variation (t-test $=-1.94, \mathrm{P}$-val$\mathrm{ue}=0.03)$ in the mean parity of women with $(0.13 \pm 0.3)$ and without $(0.32 \pm 0.7)$ endometriosis. Mean duration of infertility was not significantly varied regardless of age at menarche. However, this study showed that among women who had menarche at age
11-13.9 years, women with endometriosis were 2.10 times more likely to have primary infertility (OR=2.10, 95\% CI: 1.04, 4.23). In age at menarche of 11-13.9 years, the proportion of women with endometriosis and primary infertility was significantly higher than that of women without endometriosis but with primary infertility $\left(\chi^{2}=4.29\right.$, P-value $\left.=0.04\right)$. 
Duration of flow: It is only among women with duration of flow of 4-7 days that the proportion of women with endometriosis and primary infertility was significantly higher than that of women without endometriosis but with primary infertility $\left(\chi^{2}=6.50\right.$, P- val$\mathrm{ue}=0.01$ ). In the category of women with duration of flow of 4-7 days, those with endometriosis were 2.16 times more likely to have primary infertility (OR=2.16, 95\% CI: 1.19, 3.90) compared to those without endometriosis.

Cycle length: In the category of women who had cycle length $\geq 28$ days, the mean parity was significantly higher ( $\mathrm{t}$-test $=-2.04$,
P-value $=0.02)$ among those without endometriosis $(0.26 \pm 0.6)$ compared to those with endometriosis $(0.12 \pm 0.3)$. Although among women with cycle length $<28$ days, those with endometriosis were slightly more likely to have primary infertility $(\mathrm{OR}=1.02,95 \% \mathrm{CI}$ : $0.24,4.41$ ), among those with cycle length $\geq 28$ days, those with endometriosis were 2.34 times more likely to have primary infertility $(\mathrm{OR}=2.34$, 95\% CI: 1.32, 4.16) than those without endometriosis.

Dysmenorrhea, menorrhagia and dyspareunia among women with and without endometriosis relative to menstrual profile (Table 4).

Table 4: Parity, duration and type of infertility by menstrual characteristics and probability of endometriosis.

\begin{tabular}{|c|c|c|c|c|c|c|c|c|c|c|c|c|c|c|c|c|c|c|}
\hline \multirow{5}{*}{ Variable } & \multicolumn{6}{|c|}{ Age at menarche $<11$ yrs. } & \multicolumn{6}{|c|}{ Duration of flow $<4$ days } & \multicolumn{6}{|c|}{ Cycle length $<28$ days } \\
\hline & \multicolumn{6}{|c|}{ OR $(95 \% \mathrm{CI})=2.22(0.99,4.98)$} & \multicolumn{6}{|c|}{ OR $(95 \% \mathrm{CI})=0.49(0.09,2.74)$} & \multicolumn{6}{|c|}{ OR $(95 \% \mathrm{CI})=0.66(0.31,1.43)$} \\
\hline & \multicolumn{2}{|c|}{ Dysmenorrhea } & \multicolumn{2}{|c|}{ Menorrhagia } & \multicolumn{2}{|c|}{ Dyspareunia } & \multicolumn{2}{|c|}{ Dysmenorrhea } & \multicolumn{2}{|c|}{ Menorrhagia } & \multicolumn{2}{|c|}{ Dyspareunia } & \multicolumn{2}{|c|}{ Dysmenorrhea } & \multicolumn{2}{|c|}{ Menorrhagia } & \multicolumn{2}{|c|}{ Dyspareunia } \\
\hline & Yes & No & Yes & No & Yes & No & Yes & No & Yes & No & Yes & No & Yes & No & Yes & No & Yes & No \\
\hline & $\begin{array}{l}\text { Freq. } \\
(\%)\end{array}$ & $\begin{array}{l}\text { Freq. } \\
(\%)\end{array}$ & $\begin{array}{l}\text { Freq. } \\
(\%)\end{array}$ & $\begin{array}{l}\text { Freq. } \\
(\%)\end{array}$ & Freq. (\%) & $\begin{array}{l}\text { Freq. } \\
(\%)\end{array}$ & $\begin{array}{l}\text { Freq. } \\
(\%)\end{array}$ & $\begin{array}{c}\text { Freq. } \\
(\%)\end{array}$ & Freq. (\%) & $\begin{array}{l}\text { Freq. } \\
(\%)\end{array}$ & $\begin{array}{l}\text { Freq. } \\
(\%)\end{array}$ & $\begin{array}{l}\text { Freq. } \\
(\%)\end{array}$ & $\begin{array}{c}\text { Freq. } \\
(\%)\end{array}$ & $\begin{array}{c}\text { Freq. } \\
(\%)\end{array}$ & $\begin{array}{l}\text { Freq. } \\
(\%)\end{array}$ & $\begin{array}{l}\text { Freq. } \\
(\%)\end{array}$ & $\begin{array}{l}\text { Freq. } \\
(\%)\end{array}$ & $\begin{array}{l}\text { Freq. } \\
(\%)\end{array}$ \\
\hline $\begin{array}{l}\text { Endometri- } \\
\text { osis }\end{array}$ & $5(83.3)$ & $2(40.0)$ & $\begin{array}{c}3 \\
(100.0)\end{array}$ & $\begin{array}{c}4 \\
(50.0)\end{array}$ & $1(100.0)$ & $6(60.0)$ & $\begin{array}{c}10 \\
(100.0)\end{array}$ & 3 & 6.04 & 3.2 & 5 & 8 & 0.23 & 0.4 & 4.22 & 3.0 & $8(42.1)$ & $5(41.7)$ \\
\hline $\begin{array}{l}\text { No endome- } \\
\text { triosis }\end{array}$ & $(12.0)$ & $\begin{array}{c}3 \\
(100.0)\end{array}$ & $\begin{array}{c}10 \\
(31.3)\end{array}$ & $\begin{array}{c}3 \\
(100.0)\end{array}$ & $10(31.3)$ & $\begin{array}{c}10 \\
(76.9)\end{array}$ & 3 & 0.4 & 5.58 & 3.9 & 6 & 16 & 0.16 & 0.4 & 6.59 & 6.2 & $\begin{array}{c}11 \\
(57.9)\end{array}$ & $7(58.3)$ \\
\hline$\chi^{2}$ (P-value) & \multicolumn{2}{|c|}{$0.74(0.39)$} & \multicolumn{2}{|c|}{$0.69(0.41)$} & \multicolumn{2}{|c|}{$0.00(1.00)$} & \multicolumn{2}{|c|}{$20.07(0.000007)$} & \multicolumn{2}{|c|}{$16.43(0.00005)$} & \multicolumn{2}{|c|}{$16.43(0.00005)$} & \multicolumn{2}{|c|}{$8.92(0.003)$} & \multicolumn{2}{|c|}{$5.66(0.02)$} & \multicolumn{2}{|c|}{$3.92(0.04)$} \\
\hline \multirow[t]{3}{*}{ OR $(95 \% \mathrm{CI})$} & $\begin{array}{r}7 . \\
(0.46\end{array}$ & $\begin{array}{l}0 \\
22.70)\end{array}$ & \multicolumn{2}{|c|}{ Undefined } & \multicolumn{2}{|c|}{ Undefined } & \multicolumn{2}{|c|}{ Undefined } & Undef & ned & Unde & ined & $\begin{array}{r}16.70 \\
99.7\end{array}$ & $\begin{array}{l}2.78, \\
5)\end{array}$ & Undef & & Unde & fined \\
\hline & & Age & at menarc & he $=11-1$ & 9 yrs. & & & & Iration of $\mathrm{fl}$ & w 4-7 day & & & & & Cycle leng & $1 \geq 28$ da & & \\
\hline & & & $95 \% \mathrm{CI})=$ & $0.90(0.48$ & 1.70) & & & OR & $95 \% \mathrm{CI})=1$ & $2(0.58,2$ & & & & OR & $(95 \% \mathrm{CI})=1$ & $27(0.73$ & 2.21) & \\
\hline $\begin{array}{l}\text { Endometri- } \\
\text { osis }\end{array}$ & $\begin{array}{c}50 \\
(96.2)\end{array}$ & $\begin{array}{c}22 \\
(27.8)\end{array}$ & $\begin{array}{c}23 \\
(92.0)\end{array}$ & $\begin{array}{c}49 \\
(46.2)\end{array}$ & $7(100.0)$ & $\begin{array}{c}65 \\
(53.7)\end{array}$ & $\begin{array}{c}70 \\
(92.1)\end{array}$ & $\begin{array}{c}29 \\
(26.9)\end{array}$ & 34 (94.4) & $\begin{array}{c}65 \\
(43.9)\end{array}$ & $\begin{array}{c}10 \\
(100.0)\end{array}$ & $\begin{array}{c}89 \\
(51.1)\end{array}$ & $\begin{array}{c}71 \\
(95.9)\end{array}$ & $\begin{array}{c}29 \\
(24.0)\end{array}$ & $33(94.3)$ & $\begin{array}{c}67 \\
(41.9)\end{array}$ & $\begin{array}{c}9 \\
(100.0)\end{array}$ & $\begin{array}{c}91 \\
(48.9)\end{array}$ \\
\hline $\begin{array}{l}\text { No endome- } \\
\text { triosis }\end{array}$ & $2(3.8)$ & $\begin{array}{c}57 \\
(72.2)\end{array}$ & $2(8.0)$ & $\begin{array}{c}57 \\
(53.8)\end{array}$ & $0(0.0)$ & $\begin{array}{c}56 \\
(46.3)\end{array}$ & $\begin{array}{c}6 \\
(7.9) \\
\end{array}$ & $\begin{array}{c}79 \\
(73.1) \\
\end{array}$ & $2(5.6)$ & $\begin{array}{c}83 \\
(56.1)\end{array}$ & $0(0.0)$ & $\begin{array}{c}85 \\
(48.9)\end{array}$ & $3(4.1)$ & $\begin{array}{c}92 \\
(76.0)\end{array}$ & $2(5.7)$ & $\begin{array}{c}93 \\
(58.1)\end{array}$ & $0(0.0)$ & $\begin{array}{c}95 \\
(51.1) \\
\end{array}$ \\
\hline$\chi^{2}$ (P-value) & $56.38(0 . c$ & $0000001)$ & $15.32(0$ & 00009) & $27.91(0.0$ & 00001) & $\begin{array}{r}76 \\
(0.0000\end{array}$ & $0001)$ & $\begin{array}{r}27 . \\
(0.000\end{array}$ & $\begin{array}{l}4 \\
001)\end{array}$ & $7.22(C$ & 007) & $\begin{array}{r}92 . \\
(0.00000\end{array}$ & $\begin{array}{l}36 \\
00001)\end{array}$ & $\begin{array}{r}29 . \\
(0.0000\end{array}$ & 1 & $7.04(C$ & $.008)$ \\
\hline OR $(95 \%$ CI) & $\begin{array}{r}64 \\
(14.50,\end{array}$ & 87 & $\begin{array}{r}13 \\
(3.00,\end{array}$ & 38 & Unde & & $\begin{array}{r}31 \\
(12.46\end{array}$ & $81.04)$ & $\begin{array}{r}21 . \\
(5.03,9\end{array}$ & $\begin{array}{l}1 \\
3.71)\end{array}$ & Unde & ined & $\begin{array}{r}75 . \\
(21.98,2\end{array}$ & $86.45)$ & $\begin{array}{r}22 . \\
(5.31, \mathrm{c}\end{array}$ & & Unde & fined \\
\hline & & & e at mena & che $\geq 14$. & yrs. & & & & uration of $\mathrm{fl}$ & $w>7$ day & & & & & & & & \\
\hline & & & $95 \% \mathrm{CI})=$ & $0.36(0.12$ & 1.03) & & & OR & $95 \% \mathrm{CI})=1$ & $0(0.51,1$ & & & & & & & & \\
\hline $\begin{array}{l}\text { Endometri- } \\
\text { osis }\end{array}$ & $\begin{array}{c}26 \\
(89.7)\end{array}$ & $8(14.5)$ & $\begin{array}{c}12 \\
(100.0)\end{array}$ & $\begin{array}{c}22 \\
(30.6)\end{array}$ & $5(100.0)$ & $\begin{array}{c}29 \\
(36.7)\end{array}$ & $\begin{array}{c}1 \\
(100.0)\end{array}$ & $0(0.0)$ & $1(100.0$ & $\begin{array}{c}0 \\
(0.0)\end{array}$ & $0(0.0)$ & $\begin{array}{c}1 \\
(14.3)\end{array}$ & & & & & & \\
\hline $\begin{array}{l}\text { No endome- } \\
\text { triosis }\end{array}$ & $3(10.3)$ & $\begin{array}{c}47 \\
(85.5)\end{array}$ & $0(0.0)$ & $\begin{array}{c}50 \\
(69.4)\end{array}$ & $0(0.0)$ & $\begin{array}{c}50 \\
(63.3)\end{array}$ & $0(0.0)$ & $\begin{array}{c}6 \\
(100.0)\end{array}$ & $\begin{array}{c}0 \\
(0.0)\end{array}$ & $\begin{array}{c}6 \\
(100.0)\end{array}$ & $\begin{array}{c}0 \\
(0.0)\end{array}$ & $\begin{array}{c}6 \\
(85.7)\end{array}$ & & & & & & \\
\hline$\chi^{2}$ (P-value) & $\begin{array}{r}41 \\
(0.0000\end{array}$ & $\begin{array}{l}40 \\
00001)\end{array}$ & $17.81(0$ & 00002) & 5.41( & 02) & 1.22 & .27) & $1.22(0$ & 27) & 1.220 & .27) & & & & & & \\
\hline OR $(95 \%$ CI) & $\begin{array}{r}50 \\
(12.42,\end{array}$ & $08.71)$ & Unde & ined & Unde & & Unde & ined & Undef & & Unde & ined & & & & & & \\
\hline
\end{tabular}

Menarche: When age at menarche was $<11$ years, 11-13.9 years and $\geq 14$ years respectively, study subjects with endometriosis were 7.50 times (OR=7.50, 95\% CI: 0.46, 122.70), approximately 65 times (OR=64.77, 95\% CI: 14.50, 289.29) and about 51 times (OR=50.92, 95\% CI: 12.42, 208.71) more likely to have dysmenorrhea than those without endometriosis. When age at menarche was 11-13.9 years, those with endometriosis were approximately
14 times more likely to present with menorrhagia (OR=13.38, 95\% CI: $3.00,59.63)$.

Duration of flow: Study subjects with endometriosis and with duration of flow of 4-7 days were about 32 times more likely to present with dysmenorrhea (OR=31.78, 95\% CI: 12.46, 81.04), and about 22 times more likely to present with menorrhagia (OR=21.71, 
$95 \%$ CI: 5.03, 93.71). Among those with duration of flow of 4-7 days, the proportion of women with endometriosis who had dyspareunia was significantly higher $\left(\chi^{2}=7.22\right.$, P-value $\left.=0.007\right)$ than those without endometriosis.

Cycle length: Endometriotic women with cycle length $<28$ days were about 17 times more likely to have dysmenorrhea (OR=16.70, 95\% CI: 2.78, 99.75) than those without endometriosis. Endometriotic women with cycle length $\geq 28$ days were about 75 times
(OR=75.08, 95\% CI: $21.98,256.45)$ more likely to have dysmenorrhea and approximately 23 times (OR=22.90, 95\% CI: 5.31, 98.76) more likely to have menorrhagia than those without endometriosis. Among those with cycle length of $\geq 28$ days, the proportion of those with endometriosis who had dyspareunia was significantly higher $\left(\chi^{2}=7.04, \mathrm{P}\right.$-value $\left.=0.008\right)$ than those without endometriosis. Egg yield, matured eggs and fertilization rate among women with and without endometriosis relative to menstrual profile (Table 5).

Table 5: Egg yield, matured eggs and fertilization rate among women with and without endometriosis relative to menstrual profile.

\begin{tabular}{|c|c|c|c|c|c|c|c|c|c|c|c|c|c|c|c|c|c|c|}
\hline \multirow{4}{*}{ Variable } & \multicolumn{6}{|c|}{ Age at menarche $<11$ yrs. } & \multicolumn{6}{|c|}{ Duration of flow $<4$ days } & \multicolumn{6}{|c|}{ Cycle length $<28$ days } \\
\hline & \multicolumn{6}{|c|}{ OR $(95 \% \mathrm{CI})=2.22(0.99,4.98)$} & \multicolumn{6}{|c|}{ OR $(95 \%$ CI $)=0.49(0.09,2.74)$} & \multicolumn{6}{|c|}{ OR $(95 \% \mathrm{CI})=0.66(0.31,1.43)$} \\
\hline & \multicolumn{2}{|c|}{$\begin{array}{c}\text { Egg yield at } \\
\text { OPU }\end{array}$} & \multicolumn{2}{|c|}{ MIIs } & \multicolumn{2}{|c|}{$\begin{array}{c}\text { Fertilization } \\
\text { rate }\end{array}$} & \multicolumn{2}{|c|}{$\begin{array}{c}\text { Egg yield at } \\
\text { OPU }\end{array}$} & \multicolumn{2}{|c|}{ MIIs } & \multicolumn{2}{|c|}{$\begin{array}{c}\text { Fertilization } \\
\text { rate }\end{array}$} & \multicolumn{2}{|c|}{$\begin{array}{c}\text { Egg yield at } \\
\text { OPU }\end{array}$} & \multicolumn{2}{|c|}{ MIIs } & \multicolumn{2}{|c|}{$\begin{array}{c}\text { Fertilization } \\
\text { rate }\end{array}$} \\
\hline & Mean & $\pm s d$ & Mean & $\pm s d$ & Mean & $\pm s d$ & Mean & $\pm s d$ & Mean & $\pm s d$ & Mean & \pm sd & Mean & \pm sd & Mean & \pm sd & Mean & \pm sd \\
\hline $\begin{array}{l}\text { Endometri- } \\
\text { osis }\end{array}$ & 6.0 & 5.8 & 3.2 & 3.1 & 47.3 & 36.9 & 8.5 & 10.6 & 6.8 & 8.5 & 58.4 & 34.7 & 3.4 & 3.3 & 2.5 & 2.0 & 49.9 & 34.2 \\
\hline $\begin{array}{l}\text { No endo- } \\
\text { metriosis }\end{array}$ & 25.3 & 7.5 & 24.0 & 9.5 & 53.2 & 16.9 & 12.1 & 9.6 & 8.0 & 7.9 & 50.2 & 22.8 & 10.6 & 6.4 & 5.2 & 3.6 & 49.1 & 24.2 \\
\hline t-test & \multicolumn{2}{|c|}{-4.31} & \multicolumn{2}{|c|}{-3.70} & \multicolumn{2}{|c|}{-0.31} & \multicolumn{2}{|c|}{-0.99} & \multicolumn{2}{|c|}{-0.37} & \multicolumn{2}{|c|}{0.71} & \multicolumn{2}{|c|}{-3.90} & \multicolumn{2}{|c|}{-2.46} & \multicolumn{2}{|c|}{0.07} \\
\hline \multirow[t]{3}{*}{ P-value } & \multicolumn{2}{|c|}{0.002} & \multicolumn{2}{|c|}{0.03} & \multicolumn{2}{|c|}{0.38} & \multicolumn{2}{|c|}{0.17} & 0. & & 0.2 & & 0.0 & & 0.0 & & 0 . & \\
\hline & & Age at & ienarch & $=11-$ & 3.9 yrs. & & & Dur & on of $\mathrm{fl}$ & $v 4-7 d$ & & & & & le lengt & $\geq 28 \mathrm{~d}$ & & \\
\hline & & OR (9: & $0 \mathrm{CI})=0$ & $0(0.4$ & 1.70) & & & OR (95 & $C I)=1$ & $(0.58$ & 2.13) & & & OR (9 & $\% \mathrm{CI})=1$ & $7(0.7$ & 2.21) & \\
\hline $\begin{array}{l}\text { Endometri- } \\
\text { osis }\end{array}$ & 7.0 & 7.1 & 5.3 & 5.0 & 53.0 & 27.8 & 6.0 & 5.5 & 4.3 & 3.4 & 53.6 & 26.7 & 6.6 & 6.5 & 4.9 & 4.6 & 54.7 & 27.1 \\
\hline $\begin{array}{l}\text { No endo- } \\
\text { metriosis }\end{array}$ & 11.2 & 7.4 & 6.7 & 5.4 & 50.9 & 22.7 & 10.5 & 6.7 & 6.1 & 4.6 & 49.3 & 22.3 & 11.4 & 8.1 & 7.3 & 6.7 & 49.0 & 21.6 \\
\hline t-test & -3 . & & -1 & & 0. & & & & 2. & & 1.0 & & -4 & & -2 & & & \\
\hline P-value & 0.0 & & 0. & & 0. & & 0.000 & 00001 & 0.0 & & 0.1 & & 0.0000 & 0001 & 0.0 & & & \\
\hline & & Age : & menar & $e \geq 1$ & yrs. & & & Dur & ion of $f$ & $w>7 d$ & & & & & & & & \\
\hline & & OR (9: & $\left.{ }_{0} \mathrm{CI}\right)=0$ & $6(0)$. & 1.03) & & & OR (95 & $C I)=1$. & $(0.51$ & 1.98) & & & & & & & \\
\hline $\begin{array}{l}\text { Endometri- } \\
\text { osis }\end{array}$ & 4.8 & 3.9 & 3.6 & 3.0 & 58.6 & 28.3 & 0.0 & 0.0 & 0.0 & 0.0 & 0.0 & 0.0 & & & & & & \\
\hline $\begin{array}{l}\text { No endo- } \\
\text { metriosis }\end{array}$ & 10.5 & 7.6 & 6.3 & 5.9 & 46.6 & 21.4 & 18.8 & 11.5 & 15.3 & 13.4 & 41.4 & 10.6 & & & & & & \\
\hline t-test & -4 & & -2 & & $1 . \varepsilon$ & & & & & & 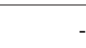 & & & & & & & \\
\hline P-value & 0.0000 & 0001 & 0.0 & & 0. & & & & & & - & & & & & & & \\
\hline
\end{tabular}

\section{Endo=endometriosis}

Menarche: The means of egg yield at ovum pickup (OPU) was most significantly noteworthy (t-test=-4.44, P-value=0.0000001) when menarche was at age $\geq 14$ years, among those with $(4.75 \pm 3.9)$ and without (10.54 \pm 7.6$)$ endometriosis. The difference in means of egg yield at OPU was less significantly noteworthy ( $t$-test $=-3.14$, P-value $=0.001)$ among those with no endometriosis $(11.19 \pm 7.4)$ than among those with endometriosis (7.03 \pm 7.1$)$ when menarche was 11-13.9 years and still less significant ( $\mathrm{t}$-test=-4.31, P-val$\mathrm{ue}=0.002)$ when age at menarche was $<11$ years among those with $(6.00 \pm 5.8)$ and without $(25.3 \pm 7.5)$ endometriosis.

Furthermore, considering study subjects who had menarche at $<11$ years, the mean of mature eggs from those without $(24.00 \pm 9.5)$ and those with $(3.17 \pm 3.1)$ endometriosis was notably varied (t-test $=-3.70, P$-value=0.03). While no statistical significance was observed among those with and without endometriosis who had menarche at age 11-13.9 years, significant difference $(\mathrm{t}$-test $=-2.59$,
P-value $=0.006)$ was observed among those with $(3.61 \pm 3.9)$ and without $(6.30 \pm 5.9)$ endometriosis. Fertilization rate was similar among women with or without endometriosis who had menarche at age $<11$ years or at age 11-13.9 years. However, fertilization rate among women with endometriosis $(58.56 \pm 28.3)$ was significantly higher ( $\mathrm{t}$-test=1.84, P-value=0.04) than among women without endometriosis (46.55 \pm 21.4$)$.

Duration of flow: At duration of flow of 4-7 days, mean egg yield at OPU was significantly higher (t-test=-4.78, P-value=0.0000001) among those without $(10.51 \pm 6.7)$ than among those with (5.98 \pm 5.5 ). Likewise, mean number of Metaphase II oocytes (MIIs or matured eggs) was significantly different (t-test=2.81, P-val$\mathrm{ue}=0.003)$ among women with $(4.30 \pm 3.4)$ and without $(6.11 \pm 4.6)$ endometriosis. There was no significant difference in fertilization rate regardless of duration of flow. 
Cycle length: Considering cycle length of $<28$ days, there was a significant difference ( $\mathrm{t}$-test $=-3.90$, P-value $=0.0003$ ) in mean egg yield at OPU in women with $(3.40 \pm 3.3)$ and without (10.56 \pm 6.4$)$ endometriosis. At cycle length of $\geq 28$ days, even stronger significant difference ( $\mathrm{t}$-test $=-4.35$, P-value $=0.00000001$ ) was observed among those with $(6.64 \pm 6.5)$ and those without $(11.41 \pm 8.1)$ endometriosis. Similarly, mean MIIs was significantly varied (t-test $=-2.46$, $\mathrm{P}$-value $=0.01)$ among those with $(2.50 \pm 2.0)$ and without $(5.22 \pm 3.6)$ the disease and equally expressively diverse ( $\mathrm{t}$-test $=-2.79$, P-value $=0.003$ ) when cycle length was $\geq 28$ days. There was no noteworthy variation in the fertilization rate among the two groups of women regardless of the cycle length.

Multivariate regression analysis (Table 6). When the three domains of menarche, number of days of menstrual flow and cycle length were considered, these three gave a significant (R2=0.9001 or $90.0 \%$, P-value $=0.00000001$ ) explanation of the difference observed in endometriosis. Menarche was more associated with endometriosis (Coef. $=0.089$, Std Err. $=0.010$, t-statistics=8.36, P-val- ue $=0.0000001,95 \%$ CI: $0.07,0.11)$ than cycle length (Coef. $=0.012$, Std Err. $=0.005$, t-statistics $=2.42$, P-value $=0.016,95 \%$ CI: 0.00, 0.02) or number of days of menstrual flow (Coef.=-0.011, Std Err.=0.026, t-statistics $=-1.42$, P-value $=0.676,95 \%$ CI: $-0.06,0.04$. When egg yield at OPU, egg quality and fertilization rate were considered as outcome of endometriosis, these three gave a significant $(86.65 \%$, P-value $=0.00000001$ ) explanation of the difference observed in endometriosis status with endometriosis being strongly positively associated with egg yield at OPU (Coef $=0.118$, Std Err=0.012, t-statistics $=10.19$, P-value $=0.00000001,95 \%$ CI: 0.09, 0.14) and with fertilization rate (Coef $=0.017$, Std Err $=0.001$, t-statistics $=17.18$, P-value $=0.00000001,95 \% \mathrm{CI}: 0.02,0.02$ ) but negatively associated with egg quality (Coef $=-0.091$, Std $\mathrm{Err}=0.017$, t-statistics $=-5.41$, P-value $=0.00000001,95 \% \mathrm{CI}:-0.12,-0.06)$. Among all the six independent variables, only menarche (Coef $=0.086$, Std Err $=0.012$, t-statistics $=7.17$, P-value $=0.00000001,95 \% \mathrm{CI}: 0.06,0.11$ ) and egg yield at OPU (Coef $=0.034$, Std Err $=0.012$, t-statistics $=2.74$, P-value $=0.007,95 \%$ CI: $0.01,0.06$ ) were significantly associated with endometriosis.

Table 6: Multivariate regression analysis with endometriosis as dependent variable and menarche, number of days of flow and cycle length as independent variables.

\begin{tabular}{|c|c|c|c|c|c|}
\hline Equation & Observed & $\mathbf{R} 2$ & $\mathbf{F}$ & P-value & \\
\hline Endometriosis & 219 & 0.9001 & 648.77 & 0.00000001 & \\
\hline Endometriosis & Coefficient & Std Err. & $\mathrm{t}$ & P-value & $95 \% \mathrm{CI}$ \\
\hline - menarche & 0.089 & 0.010 & 8.36 & 0.0000001 & $0.07,0.11$ \\
\hline - no. of days of flow & -0.011 & 0.026 & -1.42 & 0.676 & $-0.06,0.04$ \\
\hline - cycle length & 0.012 & 0.005 & 2.42 & 0.016 & $-0.00,0.02$ \\
\hline Equation & Observed & $\mathrm{R} 2$ & $\mathrm{~F}$ & P-value & \\
\hline Endometriosis & 191 & 0.8665 & 406.59 & 0.00000001 & \\
\hline Endometriosis & Coefficient & Std Err. & $\mathrm{t}$ & P-value & $95 \% \mathrm{CI}$ \\
\hline - egg yield at OPU & 0.118 & 0.012 & 10.19 & 0.00000001 & $0.09,0.14$ \\
\hline - egg quality & -0.091 & 0.017 & -5.41 & 0.00000001 & $-0.12,-0.06$ \\
\hline - fertilization rate & 0.017 & 0.001 & 17.18 & 0.00000001 & $0.02,0.02$ \\
\hline Equation & Observed & $\mathrm{R} 2$ & $\mathrm{~F}$ & P-value & \\
\hline Endometriosis & 186 & 0.9189 & 339.75 & 0.00000001 & \\
\hline Endometriosis & Coefficient & Std Err. & $\mathrm{t}$ & P-value & $95 \% \mathrm{CI}$ \\
\hline - menarche & 0.086 & 0.012 & 7.17 & 0.00000001 & $0.06,0.11$ \\
\hline - no. of days of flow & -0.007 & 0.027 & -0.24 & 0.810 & $0.06,0.05$ \\
\hline - cycle length & 0.006 & 0.005 & 1.12 & 0.265 & $-0.00,0.02$ \\
\hline - egg yield at OPU & 0.034 & 0.012 & 2.74 & 0.007 & $0.01,0.06$ \\
\hline - egg quality & -0.019 & 0.015 & -1.23 & 0.222 & $-0.05,0.01$ \\
\hline - fertilization rate & 0.002 & 0.002 & 1.03 & 0.304 & $-0.00,0.01$ \\
\hline
\end{tabular}

\section{Discussion}

Examining the risk factors for developing endometriosis is problematic. This is because the thorny issue of diagnosing endometriosis is restricted to health-facility-based studies as laparoscopy, followed by histological diagnosis are the surest and at present one of the few ways endometriosis is currently diagnosed. Houston et al., ${ }^{21}$ stated that, in hospital-based studies, assumed risk factors may actually be related more with disease detection and treatment rather than with development of disease. Also problematic is the finding of endometriosis at laparoscopy, especially without histological examination. Jansen and Russell ${ }^{22}$ contended that currently there seems to be a collective cognizance that endometriosis may manifest itself in the form of subtle lesions which may be missed by the untrained eye during laparoscopy or laparotomy. Furthermore, as stated by Nisolle et al., ${ }^{23}$ "the absence of laparoscopically detectable endometriosis does not necessarily exclude the presence of histo- 
logically detectable endometriosis." Another limitation in this study was that only five women had a BMI of $<18.5 \%$ and all of them had endometriosis.

This is the first study to examine menstrual risk factors for endometriosis among indigenous Black African women population. The findings of this study provide further support for the theories that implicate early and probably longer exposure to menstruation in the development of endometriosis. ${ }^{24,25}$ There are certain key findings in this study. First, a multivariate regression analysis indicated that endometriosis was associated with menarche, a finding that is in consonance with the report of Kvaskoff et al., ${ }^{26}$ that there were inverse relationships of endometriosis risk with menarcheal age and with the meta-analysis ofNnoaham et al., ${ }^{27}$ that there is a risk of endometriosis with early menarche but discordant with the reports of other studies..$^{27,28}$ The study of Nnoaham reported on older menarche ( $<12$ years) in contradistinction to the early age at menarche adopted in this study. ${ }^{29}$ In the current work, the probability of endometriosis was strongest among women with early $(<11$ years) menarche(OR=1.80, 95\% CI: $0.51,6.33)$ compared to those with typical (11-13.9 years) menarche (OR=1.61, 95\% CI: 0.94 , 2.74 ) or later ( $\geq 14$ years) menarche (OR=0.54, 95\% CI: $0.31,0.94)$, which agrees with Barclay's $\mathrm{s}^{30}$ and Treolar et al., ${ }^{13}$ reports that late age menarche is associated with decreased risk for endometriosis. In fact, menarche and cycle length were two out of the three menstrual characteristics in this study that had statistically significant correlation with endometriosis. Body Mass Index is a variable that has not been considered as an additional factor to the risk of endometriosis in almost all studies. The current study found that women with normal BMI who had menarche at $<11$ years old had higher probability (OR=2.81, 95\% CI: $0.25,32.16)$ for endometriosis, though the number of subjects was few. Another interesting point in the current study is the association of duration of flow with endometriosis of which the highest probability (OR=2.33, 95\% CI: 1.15 , $4.71)$ and the highest risk ( $R R=1.61,95 \% \mathrm{CI}: 1.03,2.53)$ were observed among those with normal (4-7 days) monthly menstruation, especially among overweight BMI (OR=3.14, 95\% CI: 0.94, 10.44: $\mathrm{RR}=2.13,95 \% \mathrm{CI}: 0.87,5.22$ ). This disagrees with the findings of Cramer et al., ${ }^{24}$ and Candiani et al., ${ }^{25}$ that longer monthly menstrual flow is one of the menstrual factors associated with endometriosis. One obvious limitation here is the lack of data on volume of the monthly menstrual flow and the strength of uterine contraction to support retrograde menstruation that other studies suggested to be responsible for endometriosis. ${ }^{31-35}$

In addition, this study observed that the probability and risk of endometriosis was stronger among Black African women with cycle length of $\geq 28$ days (OR=1.46, 95\% CI: $0.68,3.14$ ), most especially among women with normal BMI (OR=2.87, 95\% CI: 0.88, 9.37). This result contradicts what Grant ${ }^{36}$ and Hargrave and Abraham ${ }^{37}$ documented that women with endometriosis may have shorter menstrual cycles due to an inadequacy of corpus luteal function. Consistent with the finding of this study, Sangi-Haghpeykar and Poindexter ${ }^{38}$ concluded that long cycle length is a risk factor for endometriosis. Wei et al.,13 suggested that this conclusion from Sangi-Haghpeykar's study could have resulted from differences in the subjects' source population, race, geographical region and age which resonate with our study among indigenous Black African population geographically located in West Africa. It wasn't surprising that in this study, the proportions of endometriotic women with dysmenorrhea, menorrhagia and dyspareunia were significantly higher at normal and late age at menarche, at normal duration of menstrual flow, and predominantly at cycle length of $\geq 28$ days. Many studies have reported that dysmenorrhea, ${ }^{39-43}$ menorrhagia $^{44-46}$ and dyspareunia ${ }^{47-50}$ are closely associated with endometriosis.

Very few studies in Africa, if any at all, have reported the association of egg yield at ovum pick up, quality of eggs and fertilization rate among women with or without endometriosis relative to menstrual characteristics. This study observed that mean egg yield at ovum pick-up (OPU) was significantly lower among women with endometriosis in the three categories of menarche, most especially among those who had menarche at $\geq 14$ years. Similar observation in animal studies has been reported by Suginami et al.,51 who demonstrated that a factor inhibiting fimbrial capacity of ovum capture is present in endometriosis peritoneal fluid. Likewise, Moine et al., ${ }^{52}$ reported that endometriosis may be a predisposing factor for oocyte retrieval-induced PID. The exact mechanism of reduction in ovum pickup or in total number of eggs in endometriotic patient is not yet evidently explained, though Lenier et al.,53 and Reyburn et al., ${ }^{54}$ proposed functional NK cells which are cytotoxic effector lymphocytes that do not rearrange antigen-specific cell surface receptors, unlike cytotoxic T cells. Mean number of mature eggs was also significantly lower among endometriotic women similar to the report of Yanushpolsky et al., ${ }^{55}$ One probable mechanism for the relationship between reduced egg quality in endometriotic condition is the inflammatory milieu within the reproductive system, created by endometriosis, which is toxic to the eggs. ${ }^{56}$ Endometriosis is thus probably linked to elevated levels of cytokines and macrophages.

\section{Conclusion}

Certain menstrual symptoms were studied to examine their association with endometriosis among indigenous Black African women. As often reported, BMI was significantly lower in those without endometriosis. Dysmenorrhea, menorrhagia and dyspareunia were the most common reported symptoms among endometriotic women. Though this was not a larger study, yet it suggested thatearly menarche and long cycle length were more associated with endometriosis underpinning early exposure to menstruation as the most probable influence on the risk of endometriosis. Body Mass Index was also a factor that determined the results from this study. Egg yield and matured eggs were observed to be impacted by endometriosis while fertilization rate was not significant in those with and without endometriosis. A larger, possible nationwide, research is urgently needed to confirm and better understand these relationships.

\section{Study Limitations}

This study has certain limitations that need mentioning. The first was that very few women were in the BMI category of $<18.5$ 
$\mathrm{Kg} / \mathrm{m}^{2}$ all with endometriosis, which made it difficult to compare menstrual profile of women with and without endometriosis. Further stratification of study subjects according to menstrual profile, symptomatology and age by BMI was not done because the numbers of women in each category would have been small and analysis would not be as valid as expected. As stated earlier, by the nature of endometriosis, this study was facility-based and may not be extrapolated to the community. Though the study design was retrospective, minimal intra-person errors might have occurred in documentation of data, though nurses who were the first point-ofcontact and the gynecologists who eventually attended to the patients are experts in their fields. It is pertinent to stress that this study had some strength too, one of which was the availability of top professionals of over 20 years in the fields of clinical medicine, gynecology, nursing and embryology. The facility where this study was conducted is one of the most modern of such in Africa with up-to-date equipment, conducive environment and motivated staff.

\section{Acknowledgments}

We thank all the patients who were involved in this study and the staff of Nordica Fertility Center for their cooperation and for providing excellent facilities for this work.

\section{Funding}

None.

\section{Conflicts of Interest}

No conflict of interest is declared by all of the authors concerning the publication of this article.

\section{References}

1. Giudice LC, Kao LC. Endometriosis. Lancet. 2004;364(9447):789-799.

2. Kennedy S, Bergqvist A, Chapron C, etal. ESHRE guideline on the diagnosis and treatment of endometriosis. Hum Reprod. 2005;20(10):2698-2704.

3. Dmowski WP, Radwanska E. Current concepts on pathology, histogenesis and etiology of endometriosis. Acta Obstet Gynecol Scan (suppl). $1984 ; 123: 29-33$

4. Brosens IA, Cornillie FJ, Vasquez G, Etiology and pathophysiology of endometriosis. Prog Clin Biol Res. 1986;225:81-101.

5. Vinatier D, Orazi G, Cosson M, et al. Theories of endometriosis. Eur J Obstet Gynecol Reprod Biol. 2001;96:21-34.

6. Vercellini P, Fedele L, Aimi G, et al. Association between endometriosis stage, lesion type, patient characteristics and severity of pelvic pain symptoms: a multivariate analysis of over 1000 patients. Hum Reprod. 2007;22(1):266-271.

7. Abrao MS, Podgaec S, Dias JA, et al. Deep infiltrating endometriosis affecting the rectum and lymph nodes. Fertil Steril. 2006;86(3):543-547.

8. Podgaec S, Goncalves MO, Klajner S, et al. Epigastric pain relating to menses can be a symptom of bowel endometriosis. Sao Paulo Med J. $2008 ; 126(4): 242-244$

9. Arruda MS, Petta CA, Abrao MS, et al. Time elapsed from the onset of symptoms to diagnosis of endometriosis in a cohort study of Brazilian women. Human Reprod. 2003;18(4):756-759.

10. Batt RE, Mittwally MFM. Endometriosis from thelarche to midteens: pathogenesis and prognosis, prevention and pedagogy. J Pediatr Adolesc Gynecol. 2003;16(6):337-347.
11. Viganò P, Parazzini F, Somigliana E, et al. Endometriosis. Epidemiology and aetiological factors. Best Pract Res Clin Obstet Gynecol. 2004;18(2):177-200.

12. Hediger ML, Hartnett HJ, Louis GM. Association of endometriosis with body size and figure. Fertil Steril. 2005;84(5):1366-1374.

13. Treloar SA, Bell TA, Nagle CM, et al. Early menstrual characteristics associated with subsequent diagnosis of endometriosis. Am J Obst Gynecol. 2010;202(6):534.e1-534.e6.

14. Matalliotakis IM, Cakmak H, Fragouli YG, et al. Epidemiological characteristics in women with and without endometriosis in the Yale series. Arch Gynecol Obstet. 2008;277(5):389-393.

15. Arumugam K, Lim JM. Menstrual characteristics associated with endometriosis. Br J Obstet Gynaecol. 1997;104(8):948-950.

16. Moini A, Malekzadeh F, Amir haghmaghi E, et al. Risk factors associated with endometriosis among infertile Iranian women. Arch Med Sci. 2013;9(3):506-514.

17. Wei $\mathrm{M}$, Cheng $\mathrm{Y}, \mathrm{Bu} \mathrm{H}$, et al. Length of menstrual cycle and risk of endometriosis: A meta-analysis of 11 Case-Control studies. Medicine (Baltimore). 2016;95(9):e2922.

18. Kyama CM, Mwenda JM, Machoki J, et al. Endometriosis in African women. Women's Health. 2007;3(5):629-35.

19. Ajayi BA, Ajayi VD, Biobaku O, et al. A 10 -year study of endometriosis in an indigenous Black African population. J. Endometriosis and Pelvic Pain Disorders. 2016;8(4):157-166.

20. World Health Organization. BMI Classification. Accessed January 10, 2020.

21. Houston DE, Noller KL, Melton LJ 111, Selwyn BJ. The epidemiol- ogy of endometriosis. Clin Obster Gynecol. 1988;31:787-800.

22. Jansen RPS, Russell P. Nonpigmented endometriosis: clinical, laparoscopic and pathologic definition. Am J Obstet Gynecol. 1986;155(6):11541159.

23. Nisolle M, Paindaveine B, Bourdon A, et al. Histologic study of peritoneal endometriosis in infertile women. Fertil Steril. 1990; 53(6):984-988.

24. Cramer DW, Wilson E, Stillman R, et al. The relation of endometriosis to menstrual characteristics, smoking, and exercise. JAMA. 1986;255(14):1904-1908.

25. Candiani GB, Danesino V, Gastaldi A, et al. Reproductive and menstrual factors and risk of peritoneal and ovarian endometriosis. Fertil Steril. 1991;56(2):230-234.

26. Kvaskoff M, Bijon A, Clavel-Chapelon F, et al. Childhood and adolescent exposures and the risk of endometriosis. Epidemiology. 2013;24(2):261269.

27. Ashrafi M, Sadatmahalleh SJ, Akhoond MR, et al. Evaluation of Risk Factors Associated with Endometriosis in Infertile Women. Int J Fertil Steril. 2016;10(1):11-21.

28. Marcellin L, Santulli P, Pinzauti S, et al. Age at menarche does not correlate with the endometriosis phenotype. PLoS ONE. 2019;14(7):e0219497.

29. Nnoaham KE, Webster P, Kumbang J, et al. Is early age at menarche a risk factor for endometriosis? A systematic review and meta-analysis of case-control studies. Fertil Steril. 2012;98(3):702-712.

30. Barclay L. Late age of menarche linked to lower risk for endometriosis. Medscape. 2010.

31. Sampson JA. The development of the implantation theory for the origin of endometriosis. Am J. Obstet Gynecol. 1940;40(4):549-557.

32. Novak E. Pelvic endometriosis. Am J. Obstet Gynecol. 1931;22(6):826837. 
33. Halme J, Hammond MG, Hulka JF, et al. Retrograde menstruation in health women and in patients with endometriosis. Obstet Gynecol. 1984;64(2):151-154.

34. Gruenwald P. Origin of endometriosis from the mesenchyme of the celomic walls. Am J Obstet Gynecol. 1942;44(3):470-474.

35. Ridley JH. The histogenesis of endometriosis: a review of facts and fancies. Obstet Gynecol Surv. 1968;23(1):1-35.

36. Grant A. Additional sterility factors in endometriosis. Fertzl Steril. 1966;17(4):514-518.

37. Hargrave IT, Abraham GK. Abnormal luteal function in endometriosis. Fertil Steril. 1980;34:302-304.

38. Sangi-Haghpeykar H, Poindexter AR. Epidemiology of endometriosis among parous women. Obstet Gynecol. 1995;85(6):983-992.

39. Nasir L, Bope ET. Management of pelvic pain from dysmenorrhea of endometriosis. The Journal of the American Board of Family Practice. 2004;17(suppl 1):S43-S47.

40. Ripps BA, Martin DC. Focal pelvic tenderness, pelvic pain and dysmenorrhea in endometriosis. The Journal of Reproductive Medicine. 1991;36(7):470-472.

41. Koike H, Egawa H, Ohtsuka T, et al. Correlation between dysmenorrhea severity and prostaglandin production in women with endometriosis. Prostaglandins, Leukotrienes and Essential Fatty Acids. 1992;46(2):133137.

42. Gomibuchi $\mathrm{H}$, Taketani $\mathrm{Y}$, Doi $\mathrm{M}$, et al. Is personality involved in the expression of dysmenorrhea in patients with endometriosis? American Journal of Obstetrics and Gynecology. 1993;169(3):723-725.

43. Davis GD, Thillet E, Lindermann J. Clinical characteristics of adolescent endometriosis. Journal of Adolescent Health, 1993;14(5):362-368.

44. Sensky TE, Liu DTY. Endometriosis: associations with menorrhagia, infertility and oral contraceptives. International Journal of Gynecology and Obstetrics. 1980;17(6):573-576.

45. Tietjen GE, Conway A, Utley C, et al. Migraine is associated with menorrhagia and endometriosis. Headache: The Journal of Head and Face Pain. 2006;46(3):422-428.
46. Istre 0 , Trolle $B$. Treatment of menorrhagia with levonorgestrel intrauterine system versus endometrial resection. Fertility and Sterility. 2001;76(2):304-309.

47. Denny E, Mann CH. Endometriosis-associated dyspareunia: the impact on women's lives. J Fam Plann Reprod Health Care. 2007;33(3):189-193.

48. Orr NL, Noga H, Williams C, et al. Deep dyspareunia in endometriosis: Role of the bladder and pelvic floor. J Sex Med. 2018;15(8):1158-1164.

49. Shum LK, Bedalwy MA, Allaire C, et al. Deep dyspareunia and sexual quality of life in women with endometriosis. Sexual Medicine. 2018;6(3):224-233.

50. Yong PJ, Williams C, Bodmer Roy S, et al. Prospective cohort of deep dyspareunia in an interdisciplinary setting. J Sex Med. 2018;15(12):17651775.

51. Suginami H, Yano K, Watanabe K, et al. A factor inhibiting ovum capture by the oviductal fimbriae present in endometriosis peritoneal fluid. Fertility and Sterility. 1986;46(6):1140-1146.

52. Moini A, Riazi K, Amid V. Endometriosis may contribute to oocyte retrieval-induced pelvic inflammatory disease: Report of eight cases. Iranian Journal of Reproductive Medicine. 2004;2(1):40-42.

53. Yanushpolsky EH, Best CL, Jackson KV, et al. Effects of Endometriomas on Oocyte Quality, Embryo Quality, and Pregnancy Rates in In Vitro Fertilization Cycles: A Prospective, Case-Controlled Study. J Assist Reprod Genet. 1998;15(4):193-197.

54. Bulun SE. Ovarian endometriosis: The nemesis of eggs. Fertility and Sterility. 2014;101(4):938-939.

55. Lanier LL, Corliss B, Phillips JH. Arrousal and inhibition of human NK cells. Immunol. Rev. 1997;155:145-154.

56. Reyburn H, Mandelboim O, Vales-Gomez M, et al. Human NK cells: their ligands, receptors and functions. Immunol. Rev. 1997;155:119-125. 